\title{
The unusual multiwavelength properties of the gamma-ray source PMN J1603-4904
}

\author{
Cornelia Müller ${ }^{1,2}$, M. Kadler ${ }^{2}$, R. Ojha ${ }^{3,4}$, M. Böck ${ }^{5}$, F. Krauß ${ }^{1,2}$, G. B. Taylor ${ }^{6}$, J. Wilms ${ }^{1}$, J. Blanchard ${ }^{7}$, \\ B. Carpenter ${ }^{4}$, T. Dauser ${ }^{1}$, M. Dutka ${ }^{4}$, P. G. Edwards ${ }^{8}$, N. Gehrels ${ }^{3}$, C. Großberger ${ }^{1,2}$, H. Hase ${ }^{9}$, S. Horiuchi ${ }^{10}$, \\ A. Kreikenbohm ${ }^{2,1}$, J. E. J. Lovell ${ }^{11}$, W. McConville ${ }^{3}$, C. Phillips ${ }^{8}$, C. Plötz ${ }^{9}$, T. Pursimo ${ }^{12}$, J. Quick ${ }^{13}$, E. Ros ${ }^{14,15,5}$, \\ R. Schulz ${ }^{1,2}$, J. Stevens ${ }^{8}$, S. J. Tingay ${ }^{16}$, J. Trüstedt ${ }^{2,1}$, A.K. Tzioumis ${ }^{8}$, and J. A. Zensus ${ }^{5}$
}

${ }^{1}$ Dr. Remeis Sternwarte \& ECAP, Universität Erlangen-Nürnberg, Sternwartstrasse 7, 96049 Bamberg, Germany e-mail: cornelia.mueller@sternwarte.uni-erlangen.de

2 Institut für Theoretische Physik und Astrophysik, Universität Würzburg, am Hubland, 97074 Würzburg, Germany

3 NASA, Goddard Space Flight Center, Greenbelt MD 20771, USA

${ }^{4}$ Catholic University of America, Washington DC 20064, USA

5 Max-Planck-Institut für Radioastronomie, auf dem Hügel 69, 53121 Bonn, Germany

6 Department of Physics and Astronomy, University of New Mexico, Albuquerque NM 87131, USA

7 Departamento de Astronomía, Universidad de Concepción, 160 C Casilla, Concepción, Chile

8 CSIRO Astronomy and Space Science, ATNF, PO Box 76 Epping, Sydney NSW 1710, Australia

9 Bundesamt für Kartographie und Geodäsie, 93444 Bad Kötzting, Germany

10 CSIRO Astronomy and Space Science, Canberra Deep Space Communications Complex, PO Box 1035, ACT 2901 Tuggeranong, Australia

11 School of Mathematics \& Physics, University of Tasmania, Private Bag 37, Hobart 7001 TAS, Australia

12 Nordic Optical Telescope, Apartado 474, E38700 Santa Cruz de La Palma, Spain

13 Hartebeesthoek Radio Astronomy Observatory, 1740 Krugersdorp, South Africa

14 Observatori Astronòmic, Universitat de València, Parc Científic, C. Catedrático José Beltrán 2, 46980 Paterna, València, Spain

15 Departament d'Astronomia i Astrofísica, Universitat de València, C. Dr. Moliner 50, 46100 Burjassot, València, Spain

16 International Centre for Radio Astronomy Research, Curtin University, 6102 Perth, Australia

Received 10 October 2013 / Accepted 12 December 2013

\section{ABSTRACT}

\begin{abstract}
Context. We investigate the nature and classification of PMN J1603-4904, a bright radio source close to the Galactic plane, which is associated with one of the brightest hard-spectrum $\gamma$-ray sources detected by Fermi/LAT. It has previously been classified as a lowpeaked BLLac object based on its broadband emission and the absence of optical emission lines. Optical measurements, however, suffer strongly from extinction and the absence of pronounced short-time $\gamma$-ray variability over years of monitoring is unusual for a blazar.

Aims. In this paper, we are combining new and archival multiwavelength data of PMN J1603-4904 in order to reconsider the classification and nature of this unusual $\gamma$-ray source.

Methods. For the first time, we study the radio morphology of PMN J1603-4904 at $8.4 \mathrm{GHz}$ and $22.3 \mathrm{GHz}$, and its spectral properties on milliarcsecond scales, based on VLBI observations from the TANAMI program. We combine the resulting images with multiwavelength data in the radio, IR, optical/UV, X-ray, and $\gamma$-ray regimes.

Results. PMN J1603-4904 shows a symmetric brightness distribution at $8.4 \mathrm{GHz}$ on milliarcsecond scales, with the brightest, and most compact component in the center of the emission region. The morphology is reminiscent of a compact symmetric object (CSO). Such objects, thought to be young radio galaxies, have been predicted to produce $\gamma$-ray emission but have not been detected as a class by the Fermi $\gamma$-ray telescope so far. Sparse $(u, v)$-coverage at $22.3 \mathrm{GHz}$ prevents an unambiguous modeling of the source morphology at this higher frequency. Moreover, infrared measurements reveal an excess in the spectral energy distribution (SED), which can be modeled with a blackbody with a temperature of about $1600 \mathrm{~K}$, and which is usually not present in blazar SEDs.

Conclusions. The TANAMI VLBI data and the shape of the broadband SED challenge the current blazar classification of one of the brightest $\gamma$-ray sources in the sky. PMN J1603-4904 seems to be either a highly peculiar BL Lac object or a misaligned jet source. In the latter case, the intriguing VLBI structure opens room for a possible classification of PMN J1603-4904 as a $\gamma$-ray bright CSO.
\end{abstract}

Key words. galaxies: active - galaxies: individual: PMN J1603-4904 - radio continuum: general - gamma rays: galaxies techniques: interferometric $-\mathrm{X}$-rays: galaxies

\section{Introduction}

Blazars (flat spectrum radio quasars and BL Lac objects) are by far the largest class of Fermi/LAT detected extragalactic $\gamma$-ray sources (Nolan et al. 2012). They are a subclass of active galactic nuclei (AGN), where the angle between the line of sight and the relativistic jet is small. A more elusive class of $\gamma$-ray sources are extragalactic jets seen side-on where relativistic beaming effects are small (Abdo et al. 2010e). One sub-class of such misaligned objects are young radio galaxies, also referred to as compact symmetric objects (CSO; O'Dea 1998; Readhead et al. 1996). CSOs are not yet confirmed as $\gamma$-ray loud, however, 
theoretical models have predicted $\gamma$-ray emission from these objects (Stawarz et al. 2008; Kino et al. 2007, 2009; Kino \& Asano 2011) with expected $\gamma$-ray variability time scales substantially longer than that typically observed from blazars (Abdo et al. 2010c).

The radio source PMNJ1603-4904 (Griffith et al. 1994, PKS B 1600-489) has been classified as a low synchrotron peaked (LSP) BL Lac object (Nolan et al. 2012). Located close to the Galactic plane $\left(l=332^{\circ} .15, b=2.57\right)$, no redshift measurement has been reported to date (Shaw et al. 2013). It is associated with a bright, hard-spectrum $\gamma$-ray source detected by Fermi/LAT and called OFGL J1604.0-4904, 1FGL J1603.8-4903, 2FGL J1603.8-4904, and 1FHL J1603.7-4903, respectively (Abdo et al. 2009, 2010b; Nolan et al. 2012; Ackermann et al. 2011, 2013). The radio source lies well within the Fermi/LAT 95\%-error radius of only 0.023 (Nolan et al. 2012) and no further known radio source lies in the vicinity, making this a very high confidence association. Among the 30 brightest objects in the 2LAC-catalog (Ackermann et al. 2011), PMN J1603-4904 is one out of only two sources classified as non-variable (between $1 \mathrm{GeV}$ and $100 \mathrm{GeV}$ ) with a flux of $F_{1-100 \mathrm{GeV}}=1.29 \times 10^{-8} \mathrm{ph} \mathrm{cm}^{-2} \mathrm{~s}^{-1}$ over the first two years of Fermi/LAT observations. It was reported as non-variable with a $\mathrm{TS}_{\mathrm{var}}=39.254$, where $\mathrm{TS}_{\mathrm{var}}>41.64$ indicates a $>99 \%$-chance of variability. The second non-variable source is $4 \mathrm{C}+55.17$. This source has $\gamma$-ray properties similar to PMN J1603-4904. See McConville et al. (2011) for a discussion of the emission properties of $4 \mathrm{C}+55.17$ in the context of a CSO model. At higher $\gamma$-ray energies, PMN J1603-4904 shows a hard spectral index of $\Gamma_{10-500 \mathrm{GeV}}=1.96 \pm 0.14$ and a high flux above $50 \mathrm{GeV}$ of $S_{>50 \mathrm{GeV}}=(19 \pm 5) \times 10^{-11} \mathrm{ph} \mathrm{cm}^{-2} \mathrm{~s}^{-1}$ (Ackermann et al. 2013). A Bayesian block analysis of the $10 \mathrm{GeV}$ to $500 \mathrm{GeV}$ emission over three years of Fermi/LAT monitoring shows evidence for mild variability on longer timescales but no flaring on timescales of days to weeks (Ackermann et al. 2013; Nolan et al. 2012).

PMN J1603-4904 was observed in several large radio surveys (Griffith \& Wright 1993; Gregory et al. 1994; Massardi et al. 2011), but no high resolution observations with Very Long Baseline Interferometry (VLBI) have been reported previously. We added the source to the TANAMI ${ }^{1}$ sample in 2009 (Ojha et al. 2010; Müller et al. 2012). TANAMI is a large VLBI program, currently monitoring 84 extragalactic jets south of $-30^{\circ}$ declination at $8.4 \mathrm{GHz}$ and $22.3 \mathrm{GHz}$. The initial TANAMI sample was defined combining a radio and $\gamma$-ray selected subsample. Since the launch of Fermi, newly detected $\gamma$-ray and radio-bright extragalactic radio sources have been included in the program. For most of these sources TANAMI provides the first-ever VLBI images at milliarcsecond (mas) resolution (Müller et al. 2012, 2013).

In Sect. 2, we describe the TANAMI VLBI observations and report on the analysis of additional radio monitoring and Swift/XRT data. Then we present the results derived from first images of the milliarcsecond-scale structure of PMN J1603-4904 and its broadband spectral energy distribution (SED). In Sect. 4, we discuss the VLBI morphology and the multiwavelength properties, which are difficult to reconcile within a blazar classification scenario. We therefore also discuss alternative scenarios, including a classification as a young radio galaxy.

\footnotetext{
1 Tracking Active Galactic Nuclei with Austral Milliarcsecond Interferometry,

http://pulsar.sternwarte.uni-erlangen.de/tanami
}

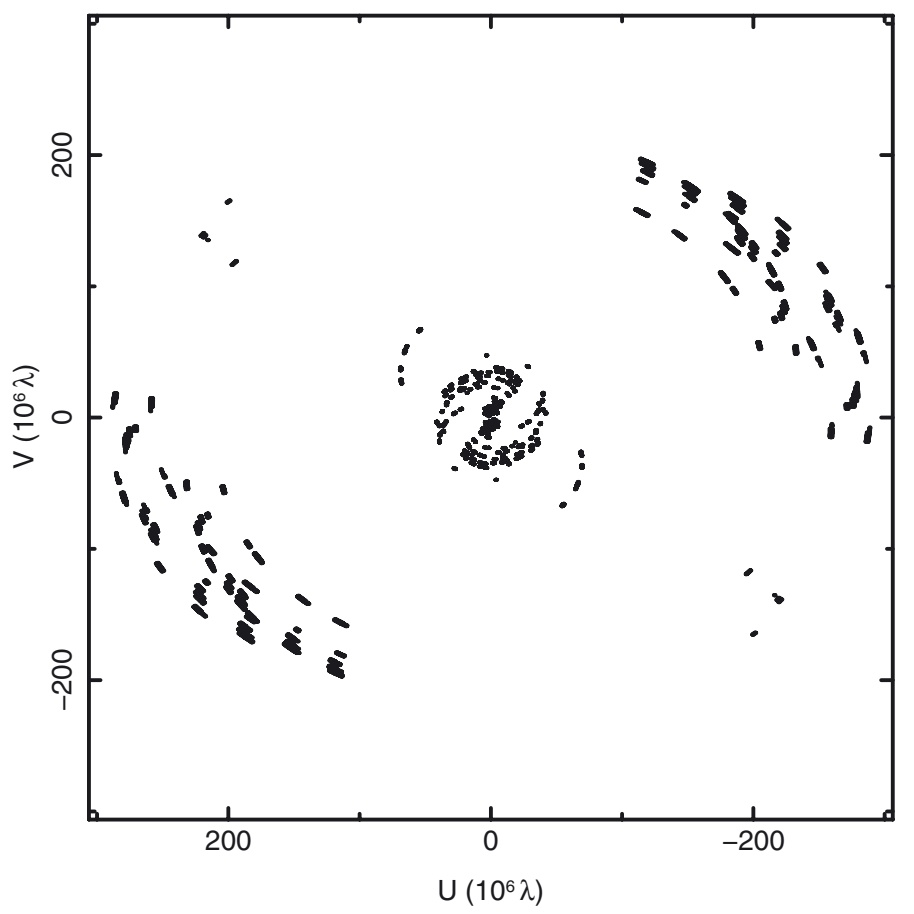

Fig. 1. $(u, v)$-coverage at $8.4 \mathrm{GHz}$ of the combined 2009 February TANAMI observations (see Table 1 for details).

\section{Observations}

\subsection{TANAMI VLBI Observations}

As part of the extended TANAMI sample (Müller et al. 2012; Böck 2012), PMN J1603-4904 has been observed three times between 2009 February and 2010 May at $8.4 \mathrm{GHz}$, including one simultaneous dual-frequency observation at $8.4 \mathrm{GHz}$ and $22.3 \mathrm{GHz}$ in 2010 May. TANAMI is using the Australian Long Baseline Array (LBA) and additional radio telescopes in Antarctica, Chile, New Zealand and South Africa (Ojha et al. 2010). Details of the observations are given in Table 1. Data were correlated on the DiFX software correlator (Deller et al. 2007; Deller et al. 2011) at Curtin University in Perth, Western Australia, and calibrated and imaged as described in Ojha et al. (2010).

Because of the different number of non-LBA telescopes in the different experiments, the $(u, v)$-coverage, array sensitivity, and angular resolution vary between the observing epochs. Figure 1 shows the $(u, v)$-coverage for the 2009 February observation at $8.4 \mathrm{GHz}$, which involved the largest number of antennas in all observations.

\subsection{ATCA radio observations}

The TANAMI sample is regularly monitored with the Australia Telescope Compact Array (ATCA). "Snapshot" observations are made at 5.5, 9, 17, 19, 38 and $40 \mathrm{GHz}$, where each frequency is the center of a $2 \mathrm{GHz}$ wide band and the flux densities are calibrated against the ATCA primary flux calibrator PKS 1934-638 and/or Uranus at $7 \mathrm{~mm}$ (Stevens et al. 2012). The primary beam widths of the ATCA radio telescope at the observed frequencies range from $10^{\prime}$ at $5.5 \mathrm{GHz}$ to $1^{\prime}$ at $40 \mathrm{GHz}$. The ATCA flux densities are found to be independent of the exact array configuration, indicating a compact nature for PMN J1603-4904 at kiloparsec scales. 
Table 1. 8.4 and 22.3 GHz TANAMI VLBI observations of PMN J1603-4904 and image parameters.

\begin{tabular}{|c|c|c|c|c|c|c|c|c|}
\hline $\begin{array}{l}\text { Date } \\
\text { yyyy-mm-dd }\end{array}$ & $\begin{array}{l}\text { Freq. } \\
{[\mathrm{GHz}]}\end{array}$ & Array configuration $^{a}$ & $\begin{array}{c}S_{\text {peak }} \\
{\left[\text { Jy beam }^{-1} \text { ] }\right.}\end{array}$ & $\begin{array}{c}\text { RMS } \\
{\left[\mathrm{mJy} \mathrm{beam}^{-1}\right]}\end{array}$ & $\begin{array}{l}S_{\text {total }} \\
{[\mathrm{Jy}]}\end{array}$ & $\begin{array}{l}b_{\text {maj }}^{c} \\
{[\mathrm{mas}]}\end{array}$ & $\begin{array}{l}b_{\min }^{c} \\
{[\mathrm{mas}]}\end{array}$ & $\begin{array}{l}\mathrm{PA}^{c} \\
{\left[{ }^{\circ}\right]}\end{array}$ \\
\hline $2009-02-23 / 27^{b}$ & 8.4 & PKS-CD-HO-MP-AT-DSS43-DS & 0.18 & 0.13 & 0.59 & 2.51 & 0.98 & 30.1 \\
\hline 2009-09-06 & 8.4 & PKS-CD-HO-MP-DSS43-TC & 0.17 & 0.23 & 0.57 & 3.36 & 1.15 & 19.2 \\
\hline 2010-05-07 & 8.4 & PKS-CD-HO-MP-AT-TC & 0.17 & 0.16 & 0.57 & 2.91 & 1.12 & 14.9 \\
\hline 2010-05-05 & 22.3 & PKS-HO-MP-AT & 0.14 & 0.35 & 0.29 & 2.09 & 1.16 & 83.9 \\
\hline
\end{tabular}

Notes. ${ }^{(a)}$ AT: Australia Telescope Compact Array (ATCA), CD: Ceduna, HO: Hobart, MP: Mopra, OH: GARS/O’Higgins, PKS: Parkes, TC: TIGO, DSS34 \& 43: Tidbinbilla (34 m \& $70 \mathrm{~m}) .{ }^{(b)}$ The two consecutive 2009 February experiments were combined, due to poorer $(u, v)$-sampling compared to the subsequent experiments at $8 \mathrm{GHz} .{ }^{(c)}$ Major, minor axes and position angle of the restoring beam.

\subsection{Optical, UV, and X-ray observations}

PMN J1603-4904 has been observed three times by the Swift satellite (Gehrels et al. 2004) between 2009 May and 2010 July for a total exposure of $6.15 \mathrm{ks}$. The Swift UV/Optical Telescope (UVOT, Roming et al. 2005) observed the source at optical/ultraviolet wavelengths with two filters (UVM2 and $U$ ). The source could not be detected at the $3 \sigma$ level and we only report upper limits on the flux, which we estimated from the background. Simultaneous to the UVOT observations, Swift X-ray Telescope (XRT, Burrows et al. 2005) observations reveal an $\mathrm{X}$-ray source which is positionally consistent with the radio source. No other archival X-ray observations are available. The Swift/XRT data were processed using the XRTDAS software package, which is part of the HEASoft package (v6.13). We reduced the data using the xrtpipeline task. The extraction of the data was performed by selecting a 47 '. 15 extraction radius centered on the source coordinates and an annulus for the background region with radii of $80^{\prime \prime}$ and $120^{\prime \prime}$. We used the accumulated data of all three observations ${ }^{2}$, which yielded a total of 77 source photons. Due to this small number statistics, we assume a standard ${ }^{3}$ absorbed power law $(\Gamma=1.64)$ and an absorption of $N_{\mathrm{H}}=6.32 \times 10^{21} \mathrm{~cm}^{-2}$ according to the Galactic HI value in this direction (Kalberla et al. 2005). The observed X-ray counts yield then a roughly estimated flux of $\sim 5 \times 10^{-13} \mathrm{erg} \mathrm{cm}^{-2} \mathrm{~s}^{-1}$ in the $0.5-10 \mathrm{keV}$ band.

PMN J1603-4904 was observed with the Gemini South telescope using the Gemini Multi-Object Spectrographs (GMOS) on 2013 March 10. The observation was made in snapshot mode in the $r^{\prime}$ band (centered at $\lambda \sim 6250 \AA$ ) to identify the optical counterpart of the radio source and to facilitate spectroscopic followup. The source was observed for $120 \mathrm{~s}$, under $0 . ' 6$ seeing and a limiting magnitude of about 25 mag was achieved.

\section{Results}

\subsection{Brightness distribution on milliarcsecond scales}

Table 1 shows the results of hybrid imaging using the CLEAN algorithm (Högbom 1974). The three 8.4 GHz VLBI observations of PMN J1603-4904 show an almost constant total correlated flux density of $570 \mathrm{mJy}$ to $590 \mathrm{mJy}$. Changes in the measured values are well below the (conservative) estimate of absolute calibration uncertainties of $\sim 20 \%$ (Ojha et al. 2010). The images have a dynamic range (ratio of peak brightness to five times the root-mean-squared noise level) of $\sim 80$ to $\sim 280$.

\footnotetext{
2 A separate X-ray data analysis of each single Swift/XRT observation was not possible.

3 For our TANAMI sample (compare also with Kadler 2005).
}

Table 2. Model fit parameters for TANAMI VLBI images.

\begin{tabular}{|c|c|c|c|c|c|c|}
\hline \multicolumn{7}{|c|}{$8.4 \mathrm{GHz}$} \\
\hline $\begin{array}{l}S^{a} \\
{[\mathrm{Jy}]}\end{array}$ & $\begin{array}{c}d^{b} \\
{[\mathrm{mas}]}\end{array}$ & $\begin{array}{l}\theta^{b} \\
{\left[{ }^{\circ}\right]}\end{array}$ & $\begin{array}{l}a_{\text {maj }}{ }^{c} \\
{[\mathrm{mas}]}\end{array}$ & $\begin{array}{l}a_{\min }{ }^{c} \\
{[\mathrm{mas}]}\end{array}$ & $\begin{array}{r}\mathrm{PA}^{c} \\
{\left[{ }^{\circ}\right]}\end{array}$ & $\begin{array}{c}T_{\mathrm{B}}{ }^{d} \\
{\left[10^{9} \mathrm{~K}\right]}\end{array}$ \\
\hline \multicolumn{7}{|c|}{ Combined 2009-02-23 \& 2009-02-27: } \\
\hline 0.17 & 2.8 & 97.0 & 2.9 & 2.9 & 138.0 & 0.35 \\
\hline 0.22 & 0.0 & - & 0.9 & 0.5 & 33.0 & 9.1 \\
\hline 0.20 & 3.4 & -79.0 & 3.0 & 3.0 & -114.0 & 0.39 \\
\hline \multicolumn{7}{|c|}{ 2009-09-05: } \\
\hline 0.16 & 2.6 & 90.0 & 2.9 & 2.9 & -155.0 & 0.34 \\
\hline 0.22 & 0.0 & - & 1.1 & 0.4 & 17.0 & 8.6 \\
\hline 0.19 & 3.3 & -77.0 & 3.2 & 3.2 & -153.0 & 0.32 \\
\hline \multicolumn{7}{|c|}{ 2010-05-07: } \\
\hline 0.17 & 2.7 & 98.0 & 3.0 & 3.0 & 177.0 & 0.35 \\
\hline 0.21 & 0.0 & - & 1.1 & 0.4 & 12.0 & 8.6 \\
\hline 0.19 & 3.2 & -78.0 & 2.9 & 2.9 & 20.0 & 0.38 \\
\hline \multicolumn{7}{|c|}{$22.3 \mathrm{GHz}$} \\
\hline$S^{a}$ & $d^{b}$ & $\theta^{b}$ & $a_{\mathrm{maj}}^{c}$ & $a_{\min }^{c}$ & $\mathrm{PA}^{c}$ & $T_{\mathrm{B}}{ }^{d}$ \\
\hline$[\mathrm{Jy}]$ & [mas] & {$\left[{ }^{\circ}\right]$} & [mas] & [mas] & {$\left[{ }^{\circ}\right]$} & {$\left[10^{9} \mathrm{~K}\right]$} \\
\hline \multicolumn{7}{|c|}{ 2010-05-05: } \\
\hline 0.04 & 2.7 & 98.0 & 3.0 & 3.0 & 177.0 & 0.01 \\
\hline 0.19 & 0.0 & - & 1.1 & 0.4 & 12.0 & 1.1 \\
\hline 0.07 & 3.2 & -78.0 & 2.9 & 2.9 & 20.0 & 0.02 \\
\hline
\end{tabular}

Notes. ${ }^{(a)}$ Integrated flux density of model component. ${ }^{(b)}$ Distance and position angle of the model component from the designated phase center. ${ }^{(c)}$ Major, minor axis extent (FWHM) of the model component and position angle of the major axis. ${ }^{(d)}$ Brightness temperature of model component.

The source is resolved in all directions and shows an eastwest orientation with three resolved distinct regions within $\lesssim 15$ mas. The brightest, most compact feature is located in the center (see Fig. 2).

In order to study the flux density and structural variability of the individual prominent jet features, we choose a simple model of three elliptical Gaussian emission components (using DIFMAP, Shepherd 1997) to fit the self-calibrated visibility data. The parameters of the model components are reported in Table 2. The central component is very small but resolved (see Kovalev et al. 2005, for details on the resolution limit). Using

$T_{\mathrm{B}}=\frac{2 \ln 2}{\pi k} \frac{S \lambda^{2}}{a_{\mathrm{maj}} a_{\mathrm{min}}}(1+z)$

where $S$ is the flux density, $a_{\text {maj,min }}$ are the major and minor axes of the component, $k$ is the Boltzmann constant, $z$ is the redshift, and $\lambda$ is the wavelength of the observation (Kovalev et al. 2005), 


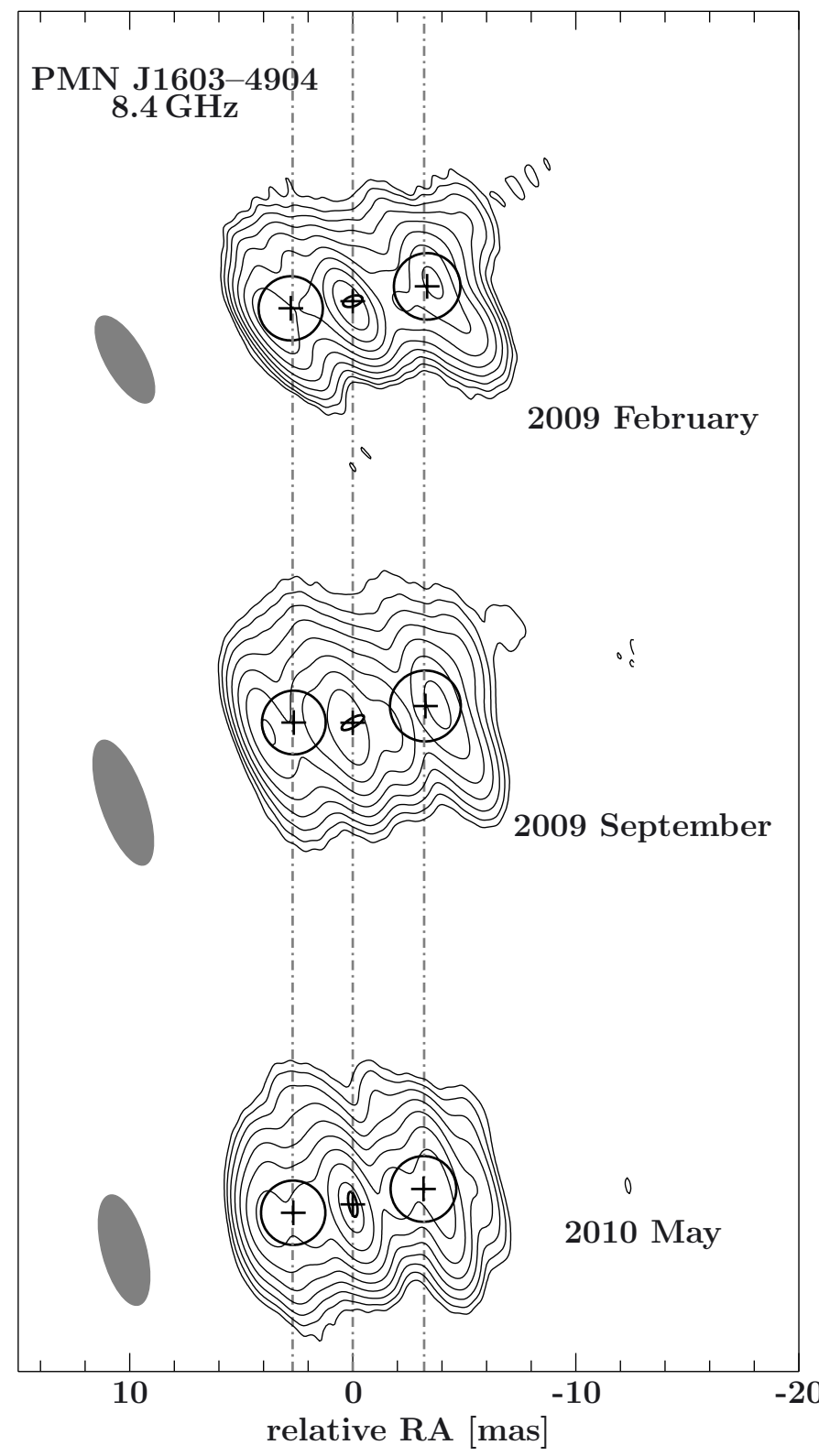

Fig. 2. Time evolution of PMN J1603-4904. CLEAN images of the first $8.4 \mathrm{GHz}$ TANAMI observations are shown. The contours indicate the flux density level, scaled logarithmically and separated by a factor of 2, with the lowest level set to the $3 \sigma$-noise-level (for more details see Table 1). The positions and FWHMs of the Gaussian emission components are overlaid as black ellipses (for model parameters see Table 2). From top to bottom: 2009 February (combined image of the 23rd and 27th), 2009 September, and 2010 May. The size of the restoring beams for each individual observation is shown as a gray ellipse on the left. Vertical dashed lines which indicate the relative positions of the eastern and western features with respect to the central component are drawn at 2.7 mas and -3.2 mas, respectively.

the brightness temperature of this brightest of all found components is $T_{\mathrm{B} \text {,central }} \gtrsim 9 \times 10^{9} \mathrm{~K}$ (due to the lack of a redshift measurement, we apply $z=0$, i.e., no redshift correction). No component shows significant flux density or brightness temperature variability over 15 months. The eastern and western regions can each be modeled with circular Gaussian flux distributions. The eastern component is about $(0.2 \pm 0.1) \mathrm{Jy}$ weaker and about 0.6 mas closer to the central component than the western one.

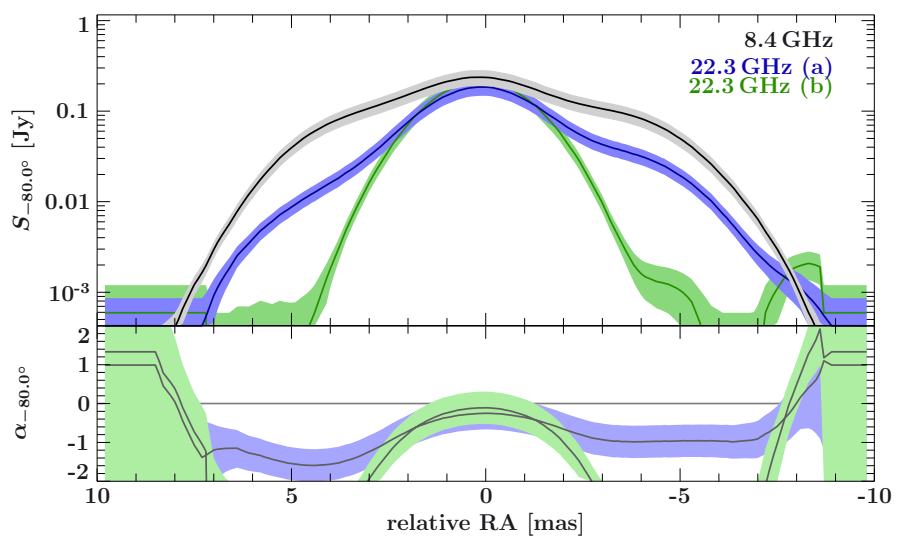

Fig. 3. Top: flux density profiles along $\mathrm{PA}=-80^{\circ}$ at $8.4 \mathrm{GHz}$ (gray) and $22.3 \mathrm{GHz}$ "extended model" (blue, a)) and "compact model" (green, b)). Bottom: spectral index along PA $=-80^{\circ}$. Displayed uncertainties correspond to a conservative estimate of absolute calibration uncertainties and on-source errors of $\sim 20 \%$. The spectral index distribution of the "extended model" is in light blue, the "compact model" is shown in light green.

The brightness temperatures of both outer components are also constant at $(3-4) \times 10^{8} \mathrm{~K}$.

To test for structural variability, we measured component positions relative to the eastern component. Within the uncertainties, no significant component motions could be found over the covered period of 15 months. Therefore we can set a limit for

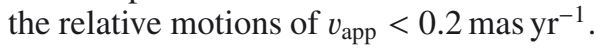

\subsection{Spectral properties on mas-scale}

In 2010 May, contemporaneous $8.4 \mathrm{GHz}$ and $22.3 \mathrm{GHz}$ TANAMI VLBI observations were performed (see Table 1). The $(u, v)$-coverage at $22.3 \mathrm{GHz}$ is poorer than that at $8.4 \mathrm{GHz}$, because the TIGO antenna is not equipped with a $22.3 \mathrm{GHz}$ receiver and the Ceduna data were not usable due to problems with the maser, such that effectively only data from four Australian antennas were available. In order to image and self-calibrate the $22.3 \mathrm{GHz}(u, v)$-data, we used the structural model found from the high-quality data at $8.4 \mathrm{GHz}$. We fixed the relative positions of the three components from the $8.4 \mathrm{GHz}$ Gaussian model and allowed only their flux densities to vary. This approach resulted in an acceptable starting model to perform amplitude self-calibration on long time scales. We then fitted the component flux densities again and performed self-calibration on iteratively smaller time scales. Overall self-calibration corrections were small and the final model was in good agreement with the original data. This model represents the most extended structure, which is still consistent with both the $(u, v)$-data at $22.3 \mathrm{GHz}$ itself and with the brightness distribution found at $8.4 \mathrm{GHz}$. More extended regions in VLBI images of extragalactic jets have steep spectra so the $22.3 \mathrm{GHz}$ emission region is unlikely to be larger than the $8.4 \mathrm{GHz}$ emission region. For these reasons, we refer to this model of the $22.3 \mathrm{GHz}$ brightness distribution as the "extended" model.

In order to estimate the spatial spectral index ${ }^{4}$ distribution, we combined the $8.4 \mathrm{GHz}$ image of 2010 May with the quasi-simultaneous $22.3 \mathrm{GHz}$ image, which was produced from the "extended model" (Fig. 3). We convolved both data sets with a common circular beam with a major axis of 3 mas and calculated cuts through the two brightness distributions along

4 The spectral index $\alpha$ is defined through $S_{v} \propto v^{+\alpha}$. 
C. Müller et al.: The unusual multiwavelength properties of the gamma-ray source PMN J1603-4904

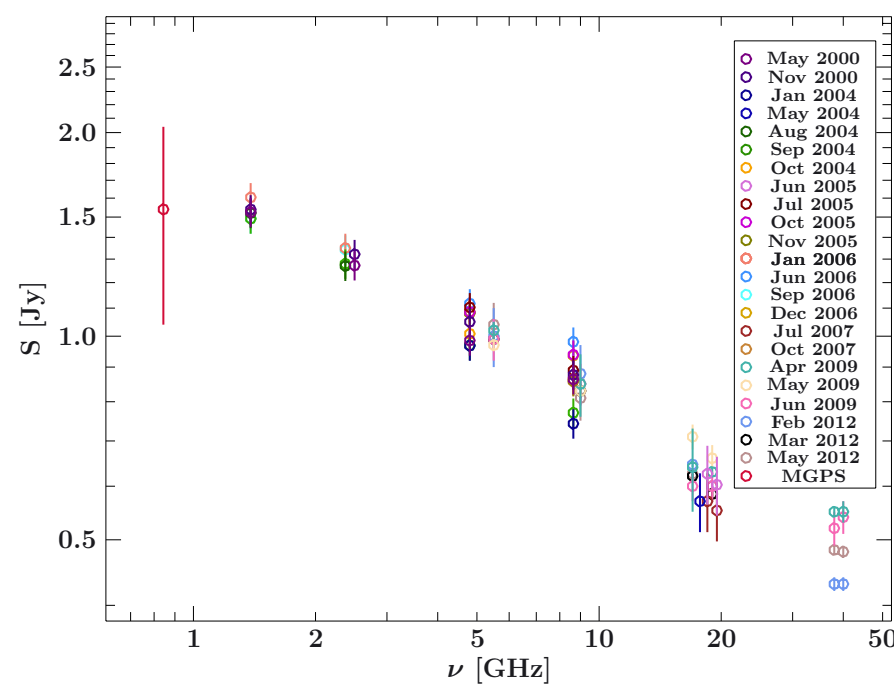

Fig. 4. Radio spectrum of PMN J1603-4904 at 6 frequencies based on the ATCA monitoring. The error bars are calculated as a frequencydependent fraction of the flux density at each frequency. The archival data of the MGPS catalog might indicate a turnover of the spectrum below $0.8 \mathrm{GHz}$.

$\mathrm{PA}=-80^{\circ}$. Formally, we can assign uncertainties to both profiles according to the absolute calibration uncertainties in both frequency bands. This approach, however, would not account for the much larger structural uncertainties at $22.3 \mathrm{GHz}$ because of the sparse $(u, v)$-coverage. To constrain these uncertainties, we derived a "compact" model of the $22.3 \mathrm{GHz}$ brightness distribution by considering only one elliptical Gaussian component in the model-fitting/self-calibration cycle described above. The resulting profile of the "compact" model is also shown in Fig. 3.

The bottom panel of Fig. 3 shows the formal spectral index profile calculated for the "extended" and "compact" models. It is obvious that the sparse $(u, v)$-coverage affects mainly the eastern and western wings of the spectral index profile. The central component is consistently found at a spectral index of $-0.75 \lesssim \alpha \lesssim-0.25$. The eastern and western regions have steep spectra in the "extended model" with $-2.0 \lesssim \alpha \lesssim-1.0$, but reach unrealistically steep values in the "compact model" $(\alpha \ll-2.0)$. We conclude that the compact model, while formally representing the sparse $(u, v)$-data well, does not lead to a self-consistent dual-frequency model.

Because absolute position information is lost in the selfcalibration process, the "compact model" would in principle allow different alignments with the $8.4 \mathrm{GHz}$ image. In particular, in a blazar scenario (see Sect. 4), one might consider the peak of the $22.3 \mathrm{GHz}$ emission in the "compact model" to be associated with the eastern source component at $8.4 \mathrm{GHz}$ (i.e., a shift of $\sim 2.7$ mas) since the tapered $8.4 \mathrm{GHz}$ image shows a potential, faint extension to the west. With this alternate alignment, the putative blazar core in the east would have a slightly inverted spectrum, but the bright $8.4 \mathrm{GHz}$ emission westward would reach again unphysical, extremely steep spectral-index values $(\alpha \ll-4)$.

These considerations reveal the central feature as the most plausible "core" of PMN J1603-4904, as the region with the flattest spectral index and the highest brightness temperature.

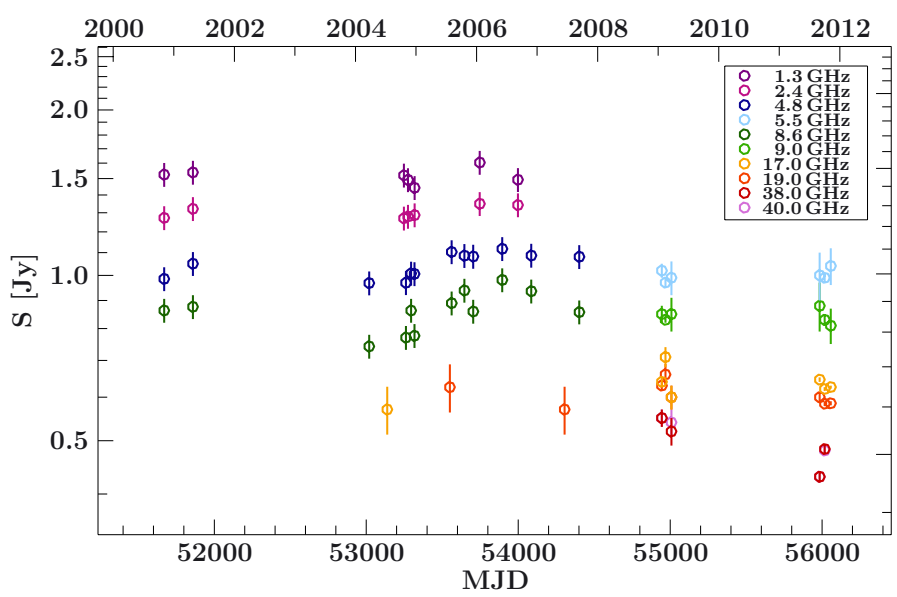

Fig. 5. Light curve of PMN J1603-4904 from the ATCA monitoring at 6 frequencies. Error bars as in Fig. 4. Note the lack of variability between 2009 and 2012. On longer timescales some evidence for lowlevel variability is seen.

\subsection{Integrated ATCA radio spectral monitoring}

In Figs. 4 and 5 we show the radio spectra and light curves which were collected as part of the C1730 ATCA program (Stevens et al. 2012) as well as of archival ATCA observations. Between 2009 and 2012 the source shows no significant variability. Its spectrum has a spectral index of $\alpha \sim-0.4$, consistent with the one determined from the integrated VLBI images $(-1.0 \lesssim \alpha \lesssim$ 0.0 , assuming the "extended model" at $22.3 \mathrm{GHz}$ ). Note, however, that the ATCA flux densities are $\sim 200 \mathrm{mJy}$ brighter. This indicates a diffuse extended emission component which is resolved out by the TANAMI VLBI array.

\subsection{Multiwavelength source association}

The association of the Fermi/LAT source 2FGL J1603.8-4904 with the radio object PMNJ1603-4904 is well established (Abdo et al. 2009, 2010b; Nolan et al. 2012). The Molonglo Galactic Plane Survey (MGPS) catalog lists the source with $S_{843 \mathrm{MHz}}=(1.54 \pm 0.5) \mathrm{Jy}$ (MGPS, source ID: J160350-490403, Murphy et al. 2007). Swift/XRT observations reveal a single, but faint, X-ray source. While the possibility of association with a spurious source within the Swift/XRT error circle cannot be ruled out, the radio position of PMN J1603-4904 clearly falls within the Swift/XRT's point spread function (PSF) of 18" half power diameter (HPD) at $1.5 \mathrm{keV}$. Combined with the favorable extrapolation of the nonthermal X-ray emission into the $\gamma$-ray regime (see Sect. 3.5 below for more details), this positional coincidence leads us to believe that PMN J1603-4904 is the most likely association of the $\mathrm{X}$-ray source.

To identify the optical/IR counterpart of PMN J1603-4904, we use data from various sky surveys. In 2010, NASA's Widefield Infrared Survey Explorer (WISE; Wright et al. 2010) mapped the sky at $3.4,4.6,12$, and $22 \mu \mathrm{m}$ (bands $W 1, W 2, W 3$, and W4) with $6^{\prime \prime}-12^{\prime \prime}$ resolution. The WISE catalog flags the associated source WISE J160350.68-490405.6 as "extended", i.e., not being consistent with a point source. Since the closest detected WISE source has a distance of $\approx 12^{\prime \prime}$ from PMN J1603-4904, the extension must be caused by a closer object that is not resolved with WISE.

Figure 6 shows the higher resolved 2MASS image (Skrutskie et al. 2006, angular resolution $\left.\sim 2^{\prime \prime}\right)$. In this $K_{\mathrm{S}}$ band 


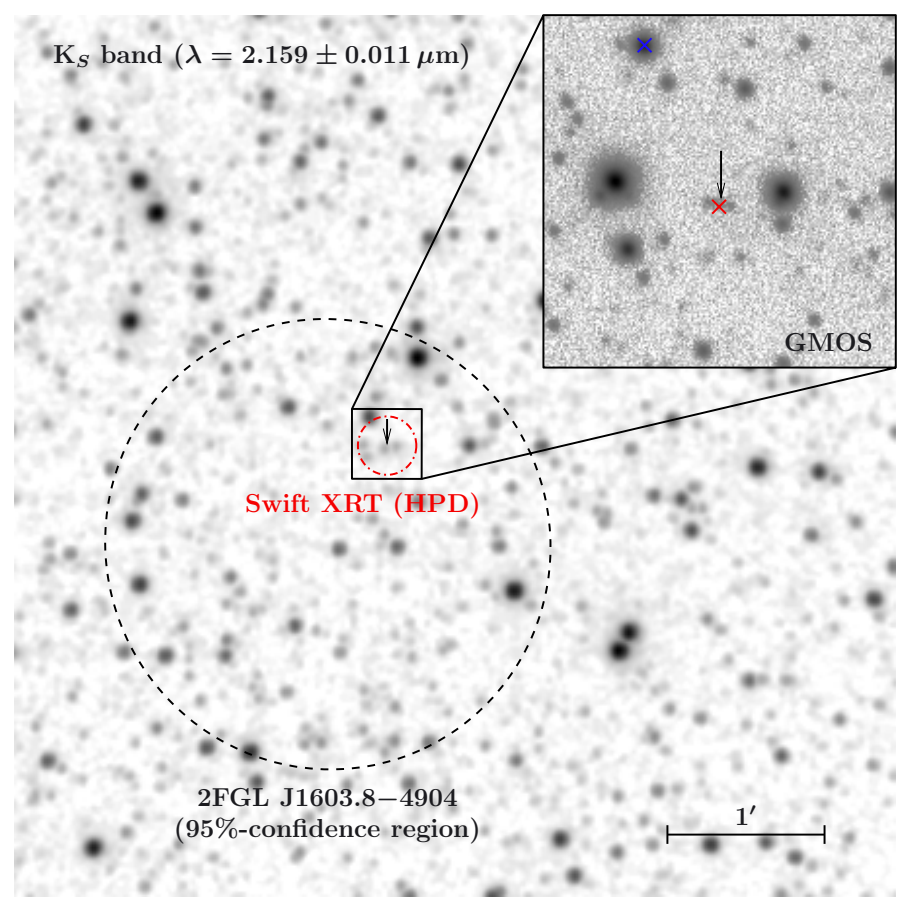

Fig. 6. Grayscale 2 MASS image ( $K_{\mathrm{S}}$ band) of the region around the radio position of PMN J1603-4904, indicated by the black arrow. The dashed black circle indicates the $95 \%$-confidence ellipse of Fermi/LAT, the dot-dashed red circle the Swift/XRT HPD. The close-up in the north western corner shows the higher resolved GMOS ( $r^{\prime}$ band) image revealing a faint elliptically shaped source. The red cross marks the position of the associated WISE/2MASS source, the blue one the closest source detected by WISE (see Sect. 3.4). No other known radio source is found in this region, indicating a high-confidence association with the high-energy counterpart.

$(2.159 \mu \mathrm{m})$ image a very faint IR source is seen which is positionally consistent and associated with PMN J1603-4904 (2MASS 16035069-4904054, ID: 655163671). This counterpart is also flagged as "photometric confused", likely due to a 4."8 distant neighboring star of comparable IR magnitude. Relatively to the counterpart to PMN J1603-4904, this object becomes fainter toward longer wavelengths. This indicates that the emission seen in WISE is probably dominated by PMN J1603-4904, however, in the following we will consider the infrared fluxes from WISE and 2MASS are contaminated, as we cannot exclude a contribution of this neighboring star in these surveys (see also Sect. 3.5).

Going to even higher resolution, the $r^{\prime}$-band GMOS observation is able to separate the neighboring star and PMN J1603-4904. The GMOS image reveals faint, extended emission with an East-West extension of 2.'5 and 1'.3 in NorthSouth direction at the position of PMN J1603-4904. The apparent magnitude of the Eastern part of the emission is about $24 \mathrm{mag}$, which we will take to be the upper limit of the point source flux density of the optical counterpart (Fig. 6).

Shaw et al. (2013) performed optical spectroscopic observations of all 2LAC BLLac objects. PMN J1603-4904 was observed (on 2009 Aug 22) with the New Technology Telescope (NTT) at La Silla Observatory. Based on low signal-to-noise spectra these authors labeled the source as a BLLac using their standard criteria and determined a limit for the redshift of $z_{\max }=4.24$. Due to its location in the Galactic plane, these optical observations suffered from strong extinction along the line of sight. Hence, more sensitive optical observations are required

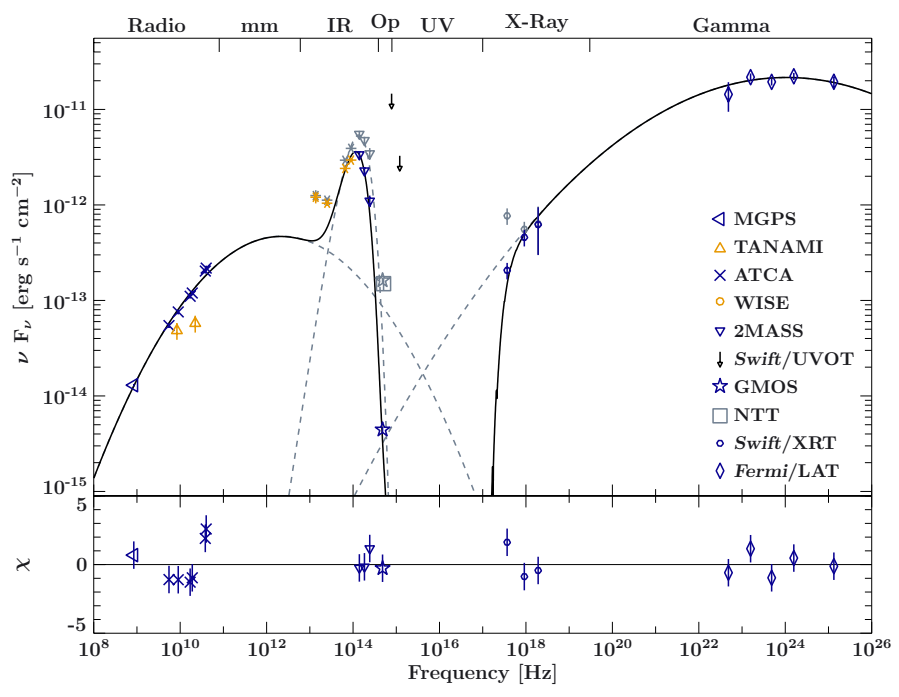

Fig. 7. Broadband $v F_{v}$ SED including total fluxes of simultaneous TANAMI observations (of 2010 May), non-simultaneous measurements in the radio by the Molonglo Galactic Plane Survey (MGPS, Murphy et al. 2007), by ATCA (Stevens et al. 2012), by WISE (Wright et al. 2010) and 2MASS (Skrutskie et al. 2006), by GMOS and NTT (Shaw et al. 2013), by Swift/XRT, UVOT, and Fermi/LAT (2FGL, Nolan et al. 2012). The data are parametrized with two logarithmic parabolas absorbed by photoelectric absorption at X-ray energies with $N_{\mathrm{H}}=1.3 \times 10^{22} \mathrm{~cm}^{-2}$ and an additional blackbody component (see Sect. 3.5 for details; $\chi^{2}=23$ for 12 degrees of freedom). Blue/Orange symbols mark the data used/ignored for the SED parametrization (solid black line) as described in Sect. 3.5. The IR and optical data are corrected for extinction (Fitzpatrick 1999; Nowak et al. 2012), and the X-ray data for absorption (Wilms et al. 2000, 2012; Verner et al. 1996), shown as corresponding gray symbols. The dashed gray lines represent the unabsorbed logarithmic parabolas as well as the extinction corrected blackbody component. The bottom panel shows the residuals of the fit in units of standard deviation of the individual data points.

to better constrain this limit and to obtain more information on the optical counterpart.

Absorption is also responsible for the absence of a matching Swift/UVOT counterpart (see Sect. 2.3). With additional positional constraints from infrared information by 2MASS (Skrutskie et al. 2006) and WISE we can exclude the two nearby UV-sources visible in the UVOT image as possible optical counterparts of PMN J1603-4904.

In the following, we assume that the reported multiwavelength counterparts are positionally consistent and discuss on this basis the broadband properties of PMN J1603-4904.

\subsection{The broadband SED of PMN J1603-4904}

We have constructed a non-simultaneous broadband SED from radio $^{5}$, optical/UV, and X-ray data, as well as published IR, optical $^{6}$, and Fermi/LAT $\gamma$-ray data (Fig. 7). Since the broadband spectral shape of PMN J1603-4904 resembles the typical double-humped SEDs of blazars, we parametrized the data with two logarithmic parabolas (Giommi et al. 2012; Chang 2010; Massaro et al. 2004), as well as the photoelectric absorption

\footnotetext{
5 For the SED parametrization, we used the ATCA rather than the VLBI data because of better frequency coverage.

6 Catalog values-corrected for extinction using standard values (see Shaw et al. 2013, for more information).
} 
component at X-ray energies ${ }^{7}$ with $N_{\mathrm{H}}=1.3 \times 10^{22} \mathrm{~cm}^{-2}$ using the tbnew model of Wilms et al. (2012) with abundances from Wilms et al. (2000) and cross-sections from Verner et al. (1996), and an extinction model for the optical (Predehl \& Schmitt 1995; Fitzpatrick 1999; Nowak et al. 2012). This results in a synchrotron peak frequency of $v_{\text {sync }} \simeq 2.2 \times 10^{12} \mathrm{~Hz}$.

As discussed in Sect. 3.4, the IR fluxes are likely to be contaminated due to source confusion, however, the contribution of the neighboring star is expected not to exceed $50 \%$ (see Fig. 6). We therefore attribute the remaining excess to PMN J1603-4904. The most striking feature in this SED is the strong excess of a factor of ten in flux in the IR band. We fit this IR excess with a black body spectrum with a temperature of $T=1662.00_{-150.0}^{+120.0} \mathrm{~K}$ to the 2MASS and GMOS data ${ }^{8}$ giving an estimate on the blackbody temperature of PMN J1603-4904 and accounting for the contribution of the neighboring star. This can most likely be associated with a dusty torus, the host galaxy (Malmrose et al. 2011), or starburst activity (see Sect. 4.2).

Given the optical source morphology, it is very unlikely that the IR excess is a foreground star: A red giant $\left(L=115 L_{\odot}\right)$ with this surface temperature would need to be at a distance of $35.7 \mathrm{kpc}$, well outside the disk of the Galaxy, while an M-dwarf with $L=2.21 \times 10^{-5} L_{\odot}$ would have to be very close at $15.7 \mathrm{pc}$. The latter is unlikely, since the absorption along the line of sight is constrained by the Galactic HI value of $N_{\mathrm{H}}=6.32 \times 10^{21} \mathrm{~cm}^{-2}$ in this direction (Kalberla et al. 2005). A nearby M-dwarf would exhibit a significantly smaller extinction.

\section{Discussion}

PMN J1603-4904 has traditionally been classified as a lowpeaked BLLac object based on its $\gamma$-ray to low-energy properties (Nolan et al. 2012) and the lack of emission lines (Shaw et al. 2013). However, the source location close to the Galactic plane makes high resolution optical spectroscopy difficult. In the following, we first review the blazar scenario before we discuss possible alternative source classifications.

\subsection{PMNJ1603-4904 as a BL Lac-type object}

Within the unification scheme of radio-loud AGN (Antonucci 1993; Urry \& Padovani 1995), BL Lac objects are considered to be the beamed version of FR I type galaxies (Fanaroff \& Riley 1974), with jets pointing close to the line of sight. The broadband SED can locally be well-described by a power law component. Thermal emission features in the optical and infrared are usually outshined by the non-thermal jet emission (e.g., Abdo et al. 2010a; Chen \& Shan 2011; Plotkin et al. 2012). It is only in very few blazars that an IR excess over the underlying power

\footnotetext{
7 Note, that the amount of absorption is not well constrained because of the low photon statistics in the X-ray band and the oversimplified continuum model. Furthermore, as the source is located in the Galactic plane, the high $N_{\mathrm{H}}$ value can be explained by ionized and/or molecular gas not measured by HI observations of the Leiden/Argentine/Bonn Survey (LAB, Kalberla et al. 2005).

8 As the NTT data were already corrected for extinction using a different correction factor, only the GMOS data were used for the parametrization in this work. The WISE data were excluded in the parametrization, due to poorer angular resolution compared to 2MASS, declining toward longer wavelengths of the WISE bands (6"' 1,6 '. 4 , $6 .{ }^{\prime \prime} 5,12^{\prime \prime} 0$ at $3.4,4.6,12$, and $22 \mu \mathrm{m}$, respectively, Wright et al. 2010). Nevertheless, the WISE data match this parametrization of the SED, further indicating that PMN J1603-4904 is dominating the emission in this band.
}

law component can be related to a hot torus (Malmrose et al. 2011).

BL Lacs are in general strongly variable across the whole electromagnetic spectrum and show a clear double-humped SED shape. Based on the position of the synchrotron hump, subclassifications are made into low- (LSP), intermediate- (ISP) and high-synchrotron-peaked (HSP) BL Lacs with $v_{\text {peak }}^{S}<10^{14} \mathrm{~Hz}$, $10^{14}<v_{\text {peak }}^{S}<10^{15} \mathrm{~Hz}$ and $v_{\text {peak }}^{S}>10^{15} \mathrm{~Hz}$, respectively (e.g., Donato et al. 2001). High angular resolution VLBI measurements of BL Lacs in general reveal highly polarized $(\sim 10 \%)$ jet emission (Lister \& Homan 2005; Hovatta et al. 2010; Linford et al. 2011) and apparent superluminal motion of individual jet components (Kellermann et al. 2004; Lister et al. 2009). Multifrequency VLBI observations (e.g., Taylor et al. 2005) of mas-scale blazar jets show that the radio spectral index distribution of the core is flat to inverted, i.e., self-absorbed while outer jet emission becomes optically thin.

Within the blazar-classification scenario, multiple observational facts of PMNJ1603-4904 point to a highly peculiar object:

Variability: multiwavelength monitoring of PMN J1603-4904 reveals no short-term or rapid flaring variability. After two years of Fermi/LAT monitoring, in the 2FGL catalog (Nolan et al. 2012), PMN J1603-4904 was listed as a non-variable blazar-like object. However, recently, a low, long-time variability at $\geq 10 \mathrm{GeV}$ was reported (Ackermann et al. 2013). No major outburst at $\gamma$-rays was seen in PMN J1603-4904, in contrast to most other blazars (Abdo et al. 2010c). This lack of short-term variability over years and across the electromagnetic spectrum is generally unusual, although Fermi/LAT monitoring shows that blazars can stay in non-flaring states over long periods (Abdo et al. 2010c). A similar stable behavior of PMN J1603-4904 is seen at radio wavelengths. The multifrequency ATCA light curve shows no significant variability over $\sim 4$ years (see Fig. 5). Note, however, that long-term radio monitoring at mm-wavelengths (Hovatta et al. 2007) determined variability time scales of AGN of about $~ 4$ years. Archival ATCA data of PMN J1603-4904 going back about a dozen years, however, show some evidence for low-level, long timescale flux density variation similar to that seen in some GPS and CSS sources (Tingay et al. 2003). At milliarcsecond resolution, the TANAMI VLBI observations show no structural or flux density variability over a period of $\sim 15$ months (see Sect. 3 ). No fast motion of jet components was detected, which is uncommon for $\gamma$-ray bright, highly beamed jets (Lister et al. 2011). However, Piner et al. (2010) found that TeV blazars can exhibit undetectable parsecscale motions despite being very strong in $\gamma$-rays. Note that the TeV blazars are generally HSPs while PMN J1603-4904 has a synchrotron peak frequency below $10^{14} \mathrm{~Hz}$.

Polarization: for PMN J1603-4904, observations by Murphy et al. (2010) put a limit on the overall polarization of $<1.2 \%$ at $20 \mathrm{GHz}$ and $<1 \%$ at 5 and $8.4 \mathrm{GHz}$. A higher degree of polarization on smaller angular scales cannot be excluded and might be detected by polarization measurements at higher angular resolution, as the VLBI observations presented here do not include polarimetry.

Broadband spectral properties: the $\gamma$-ray spectral slope of PMN J1603-4904 (see Sect. 1) matches the ones of the luminous, hard-spectrum Fermi/LAT AGN: PMN J1603-4904 
(with $\left.\Gamma_{100 \mathrm{MeV}-100 \mathrm{GeV}}=2.04 \pm 0.04\right)$ is among the 30 brightest 2LAC objects (Ackermann et al. 2011) which have a mean $\gamma$-ray spectral index of $\Gamma_{100 \mathrm{MeV}-100 \mathrm{GeV}} \approx 2.0$. With the exception of the radio galaxy NGC 1275 all of these 30 brightest sources are classified either as flat spectrum radio quasars (FSRQs) or as BL Lacs.

Based on its infrared colors, WISE J160350.68-490405.6 (the counterpart associated with PMN J1603-4904) would be consistent with the definition of the "WISE gamma-ray strip" for BL Lacs (Massaro et al. 2011, 2012). However, the "WISE blazar strip" is defined based on sources at high Galactic latitudes and all projections in the WISE color space were treated separately, while D'Abrusco et al. (2013) modeled and selected the "WISE locus" based on the Principal Component space generated by the whole three-dimensional WISE color distribution corrected for Galactic extinction of confirmed $\gamma$-ray blazars. Using the extinction-corrected colors ${ }^{9}$ instead and applying the method described in D'Abrusco et al. (2013), the source is not a candidate blazar within the definition the 3D-WISE locus (R. D'Abrusco, priv. comm.).

In a typical BLLac, the non-thermal jet emission would outshine all other broadband emission components and the infrared component is expected to be primarily due to synchrotron processes. Contrary to this, the broadband SED of PMN J1603-4904 shows a strong excess in the infrared and the broadband parametrization requires an additional blackbody

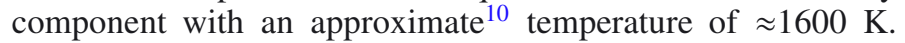
Such infrared emission features are not expected to show up in BL Lac SEDs (Abdo et al. 2010a).

The broadband SED parametrization (Fig. 7) shows a striking Compton dominance, the high energy emission hump is about two orders of magnitude above the synchrotron peak, which further challenges the BL Lac classification scenario ${ }^{11}$. The double-humped broadband emission of BLLacs is generally well described by synchrotron-self Compton models (e.g., Ghisellini et al. 2010), and such a dominance at high energies would then lead to the "Compton catastrophe". As long as no external radiation fields are considered, this particular broadband feature of PMN J1603-4904 is difficult to reconcile with the BL Lac classification. Broadband emission models including external photons from the accretion disk, the broad line region or the cosmic microwave background (e.g., Dermer et al. 2009; Finke 2013; Potter \& Cotter 2013) could explain the SED, but are more commonly used for flat spectrum radio quasars, and would hence be disfavored for this source due to the lack of optical emission lines or thermal disk emission.

Milliarcsecond structure: our TANAMI dual-frequency VLBI observations reveal a resolved and symmetric brightness distribution on milliarcsecond scales. The brightest component in the center shows a flat spectrum and the highest brightness temperature. This result is in contrast to the typical BL Lac structure of an optically thick core component as the bright end of an one-sided jet, where the spectral index changes from flat or inverted to steep further downstream (e.g., Taylor et al. 2005). The

\footnotetext{
9 Extinction-corrected colors are derived from our broadband SED parametrization (see Sect. 3.5).

${ }^{10}$ As discussed Sect. 3.4, we cannot exclude that the reported WISE fluxes of PMNJ1603-4904 are contaminated by the emission of a neighboring source.

${ }^{11}$ However, note that the peak flux of the simple, analytical parametrization with two logarithmic parabolas is not well constrained in the low energy regime.
}

brightness temperature of the three components of $T_{\mathrm{B}} \leq 10^{10} \mathrm{~K}$ is much lower than comparable values of blazars, i.e., of beamed emission (Ojha et al. 2010).

Due to the limited $(u, v)$-coverage at $22.3 \mathrm{GHz}$, no unique well-defined model of the brightness distribution at this frequency could be derived. As described above, we tested different extreme models and image alignments within the ranges allowed by the visibility data. No other alignment resulted in a physical representation of the spectral index distribution along the jet (see Sect. 3.2).

The comparison of our TANAMI results with flux density measurements of ATCA reveals that the VLBI observations miss $\sim 20 \%$ of the total flux density at $8.4 \mathrm{GHz}$. If PMN J1603-4904 is indeed a BLLac object, this difference could be explained by halo emission equivalent to the top-view of FR I lobes (e.g., Giroletti et al. 2004; Kharb et al. 2010) which might be detected with deeper ATCA observations.

In conclusion, the variability, polarization, and its broadband SED, as well as the VLBI structure and spatial spectral distribution of PMN J1603-4904 appears very atypical compared to blazar properties.

\subsection{Alternative classification scenarios}

The peculiarity of PMN J1603-4904 regarding its classification as a BL Lac type object leaves room for alternative interpretations of the multiwavelength data. In the following we discuss several possible scenarios in an attempt to get a better description of the observed broadband properties of PMN J1603-4904.

PMN J1603-4904 as a Galactic source: the source location close to the Galactic plane, the lack of a redshift measurement, and its non-thermal broadband emission might suggest a possible Galactic origin. The very low variability at high energies, however, combined with the lack of periodicity, and the non-detection of an optical galactic companion excludes the possibility that PMNJ1603-4904 is a black hole binary system. We also considered the unlikely classification as a pulsar wind nebula (PWN) even though no matching pulsar is known. Based on its VLBI properties we can easily exclude this scenario: the radio luminosity of PMN J1603-4904 is much higher than usually measured for PWNe (e.g., Frail \& Scharringhausen 1997). Moreover, assuming that the VLBI structure of $\sim 15$ mas corresponds to an extension of a few parsec, which is the mean PWN extension (Gaensler \& Slane 2006), would place PMN J1603-4904 well outside the Galaxy at a distance of a few Mpc.

Symmetric mas-scale morphology caused by gravitational lensing: as PMN J1603-4904 shows a striking symmetry on mas scales, both in structure and flux density distribution, the possibility of seeing a gravitationally lensed object should be considered. Assuming that this symmetric VLBI structure is influenced by lensing causing fainter doubles of the lensed source within a region of $\sim 15$ mas, the corresponding Einstein radius would be on the order of several milliarcseconds. It could also be larger, assuming that the lens is located north/south of the source, i.e., not the full ring is seen but only a part of it (yielding the observed elongation). Due to the constant and stable multiwavelength behavior over months to years, we can exclude a lens of a few solar masses in the parsec regime. Moreover, Wilkinson et al. (2001) found that lensing by intergalactic massive objects 
in the mas-regime is highly unlikely. The lensed system with the smallest separation known to date is S3 $0218+35$ with 335 mas (Biggs et al. 1999), while other gravitational lensed systems are beyond VLBI scales. While we cannot fully exclude a more massive object $\left(M_{\text {lens }} \gtrsim 10^{6} M_{\odot}\right)$ located at a distance of a few Mpc causing lensing on mas-scales based on our data, we consider it to be very unlikely due to the non-detection of a bright optical source within the line of sight. More exotic lenses with masses around $10^{2-5} M_{\odot}$ at shorter distances (such as intermediate-mass black holes in the Galactic halo or beyond) are unlikely.

PMNJ1603-4904 as an edge-on jet system; the $8.4 \mathrm{GHz}$ VLBI structure of PMN J1603-4904 suggests a double-sided source like in FRI radio galaxies, but on smaller scales, i.e., a jet-counterjet system seen side-on. The intrinsic symmetry and especially the compactness with a small angular separation could indicate a young, not yet evolved, radio galaxy. In the following we discuss in particular how the multiwavelength data of PMN J1603-4904 are in agreement with general properties of CSOs.

The similarity to the double-sided morphologies of presentday radio galaxies has thus led to the hypotheses that these objects are either the young versions of their larger FR I/FR II counterparts (Fanaroff \& Riley 1974; Readhead et al. 1996; Fanti et al. 1995) or "frustrated" jets, i.e., confined due to interaction with a dense, surrounding medium (e.g., Bicknell et al. 1997; Carvalho 1998). However, the "evolution" or "youth" scenario is more commonly accepted and further supported by kinematic measurements of the hot spots revealing characteristic ages less than $10^{3}$ years (e.g., Owsianik \& Conway 1998; An et al. 2012). Contrary to flat blazar spectra, the radio spectra of these compact objects show a peak at $\sim 1 \mathrm{GHz}$ or even a steep spectrum, leading to classification as a Gigahertz-peaked source (GPS) or compact steep spectrum source (CSS), respectively. CSOs are characterized by a distinct double structure with an intensity ratio of less than 10:1 and similar steep spectra and a central, flat-spectrum component (Peck \& Taylor 2000; Sokolovsky et al. 2011). They can be distinguished from other radio-loud AGN based on their low radio variability (typically $<10 \%$ over timescales of years; Fassnacht \& Taylor 2001) and their low radio polarization $(\leq 1 \%$; Peck \& Taylor 2000). Due to the high jet inclination angle little to no motion of individual jet components is observed (with very few exceptions; Owsianik \& Conway 1998; Taylor et al. 2009). Since short-time variability is linked to relativistic beaming, we expect that young radio galaxies show at most only mild broadband variability.

The possibility of $\gamma$-ray emission from CSO objects has been considered due to inverse-Compton up-scattering of surrounding photon fields by the lobe electrons (Stawarz et al. 2008; Orienti et al. 2011) and based on that, the first set of Fermi/LAT sources has been investigated (McConville et al. 2011), but no unambiguous detection of a $\gamma$-ray loud CSO has been confirmed, yet. As a class, CSOs are important sources to probe the origin of high-energy emission in active galaxies and to study the interaction of jets with the ambient medium in the nuclear region.

The high-resolution TANAMI images of PMN J1603-4904 at $8.4 \mathrm{GHz}$ show mas-scale structure in agreement with a CSO morphology. This structure, the low $T_{\mathrm{B}}$ values, and the steep spectral index distribution of the outer components may indicate a very high jet inclination angle. In such a case, at most moderate relativistic beaming is expected. This possible classification is further supported by only mild long-time multiwavelength variability (Fig. 5), the lack of apparent superluminal motion and high-energy flares, and the stability in flux density and size of the individual jet components. The morphologies of most CSOs show a relatively faint core with respect to the lobe emission (Taylor et al. 1996). If we see PMN J1603-4904 at a large inclination angle, the prominent and putative most active feature would be the central component, which could explain the observed mild variability. Usually CSOs are dominated by their steep spectrum lobes but the greater contribution from the core with its flatter spectrum here could be flattening the overall radio spectrum, resulting in the observed spectral index of $\alpha=-0.4$. In particular, the detected brightness temperatures and the overall radio spectral index of PMN J1603-4904 is consistent with mean values for CSOs of $T_{\mathrm{B} \text {,mean }} \sim 10^{9} \mathrm{~K}$ and $\alpha_{\text {mean }}=-0.52$ (between $2.3 \mathrm{GHz}$ and $8.4 \mathrm{GHz}$ ) reported by Sokolovsky et al. (2011), lower than values for bright beamed core-jet sources (Kovalev et al. 2005; Ojha et al. 2010). Due to very sparse frequency coverage below $1 \mathrm{GHz}$, our ATCA observations are not sufficient to probe a possible spectral turnover as seen in CSO/GPS sources, though the archival MGPS data could indicate a GHz-peak. The archival measurements by Murphy et al. (2010) give upper limits to the overall polarization of $\leq 1.2 \%$ further supporting the CSO classification.

Our kinematic analysis of three VLBI observation epochs only results in an upper limit on the apparent jet speed, requiring further monitoring to check for significant motion in the opposite direction with respect to the central feature in order to determine the age of the radio source. However, assuming axis symmetry and a jet orientation close to the plane of the sky and using the modelfit parameters (see Table 2), the flux ratio of the two extended features gives $R \approx 1.2$, i.e., a corresponding jetto-counterjet surface brightness ratio. Hence, jet and counterjet exhibit a similar Doppler factor. For other sources in the plane of the sky, e.g., NGC 1052 (Vermeulen et al. 2003), a pattern speed of $\beta \approx 0.25$ is measured. Together with the assumption of optically thin emission with $\alpha=-0.5$ and using

$R=\left(\frac{1+\beta \cos (\theta)}{1-\beta \cos (\theta)}\right)^{3-\alpha}$,

this results in an inclination angle of $\theta \sim 84^{\circ}$. Since both, $\beta$ and $\alpha$ are rather conservative assumptions, it is unlikely that the inclination angle is smaller.

The intriguing double-sided, symmetric $8.4 \mathrm{GHz}$ emission is confined to an extension of less than 15 mas. In standard cos$\operatorname{mology}\left(H_{0}=73 \mathrm{~km} \mathrm{~s}^{-1} \mathrm{Mpc}^{-1}, \Omega_{\mathrm{m}}=0.27, \Omega_{\lambda}=0.73\right)$, the redshift-dependent ratio between angular size and linear extension peaks at $z \sim 1.6$. As a consequence the linear diameter of PMN J1603-4904 cannot be larger than $d_{\text {limit }} \lesssim 125$ pc, placing the VLBI resolved structure well below the theoretical limit of CSO extensions. There is evidence for a western extension of the mas-scale jet seen in the $8.4 \mathrm{GHz}$ VLBI data and we find a discrepancy between TANAMI and ATCA flux density measurements at $8.4 \mathrm{GHz}$ of $\sim 20 \%$. This could indicate radio emission at larger scales. Although unexpected for young radio sources, such extended emission in association with a young double source has been reported in the literature (Baum et al. 1990; Tingay et al. 2003) and proposed to be due to recurrent activity in these objects with the extended emission attributed to past epochs of activity. Edwards \& Tingay (2004, their Fig. 9) suggest that such "missing" flux between ATCA and VLBI baselines is more common for a CSS than for a GPS source. ATCA observations at $5.5 \mathrm{GHz}$ and $9 \mathrm{GHz}$ show no extended emission, confirming the compact overall structure of this source down to $\sim 1^{\prime}$. 
Starburst-like broadband emission: in general the SED resembles the broadband emission of starburst galaxies (Lenain et al. 2010), where the enhanced IR emission is due to enhanced star formation activity. One expects temperatures on the order of a few tens of kelvins for dust emission (Elbaz et al. 2011) while higher temperatures are explained by enhanced star emission. Nuclear temperatures around $(1-2) \times 10^{3} \mathrm{~K}$ are found in Type II Seyferts (Lira et al. 2013).

Four starburst galaxies are also detected in $\gamma$-rays by Fermi/LAT (Abdo et al. 2010d; Ackermann et al. 2011), M 82 and NGC 253 even up into the TeV range (Acciari et al. 2009; Acero et al. 2009). For this source type, the origin of the $\gamma$-ray emission is explained by interactions of cosmic rays with interstellar gas rather than due to AGN activity. At these high energies, starburst galaxies show no significant variability with spectral indices in the range of $\Gamma_{\gamma}=2.1-2.4$ (Ackermann et al. 2012). In contrast, PMN J1603-4904 shows a mild variability on longer timescales in the $\gamma$-rays (Ackermann et al. 2013).

Moreover, the $\gamma$-ray fluxes of these starburst galaxies are in the range of $(5-11) \times 10^{-10} \mathrm{ph} \mathrm{cm}^{-2} \mathrm{~s}^{-1}$ (Ackermann et al. 2011). These fluxes are about ten times fainter than the reported $\gamma$-ray flux of PMN J1603-4904, which is the most important caveat for this interpretation. Assuming a similar $\gamma$-ray luminosity for all sources of this class and assuming the $\gamma$-ray emission of PMN J1603-4904 is only due to starburst activity, this would give a distance approximately three times closer than these $\gamma$-bright starburst galaxies $(\sim 1-4 \mathrm{Mpc}$, comparable to the Andromeda galaxy or Centaurus A). Despite high extinction and high source density along this line of sight, it is very unlikely that such a close object would have been missed by multiwavelength observations until now. This constraint is a strong argument against the starburst interpretation of the SED, but only if no $\gamma$-rays are emitted by an additional AGN or jet. These constraints require further statistics on the $\gamma$-ray properties of starbursts.

Lenain et al. (2010) report on the $\gamma$-ray emission of radioloud starburst galaxies and conclude that the parsec-scale jet of NGC 1068 shows a significant contribution to the high-energy emission. These findings could explain the long-term $\gamma$-ray variability and the higher flux compared to other $\gamma$-ray bright starbursts by a significant influence of the AGN. This is further supported by the high core dominance, suggesting that the center is the putative most active region if we really see the source at a large inclination angle, as discussed in the previous paragraph.

Though better multiwavelength coverage is required to precisely interpret the SED, it is striking that the IR emission, the $\gamma$-ray spectral index and its low (long-term) variability are in good agreement with starburst broadband properties. If further optical and IR observations confirm starburst activity, then PMN J1603-4904 would be the $\gamma$-ray brightest object in this particular source class.

Observations of radio-loud starbursts show evidence for a CSO-starburst connection. Tadhunter et al. (2011) find that radio-loud starburst galaxies tend to show morphologies unlike typical FR I/II-class objects. Compact radio sources $(d<15 \mathrm{kpc})$ are found to have a significantly higher star formation rate than more evolved sources, however, a possible selection effect must be considered (Dicken et al. 2012). Hence, no simple interpretation of merger-induced star formation triggering jet activity can generally be applied.

According to the available multiwavelength data and keeping in mind the most limiting factor, the lack of well constrained optical spectra, this classification scenario gives an intriguing picture of the possible nature of PMN J1603-4904. The $\gamma$-ray emission could be explained by a contribution of the starburst activity but definitely requires an additional (dominating) spectral component to model the contribution of the AGN emission to compensate for the disagreement in brightness and long-term variability not seen in $\gamma$-ray bright starburst galaxies. This alternative interpretation of the broadband emission would be more plausible, but contributions of the young radio jet as proposed by Stawarz et al. (2008) should still be considered.

\section{Summary and conclusions}

We have presented the first VLBI observations and additional multiwavelength data for the $\gamma$-ray bright source PMN J1603-4904. It exhibits multiwavelength properties atypical for well-established $\gamma$-ray emitting object classes. We have discussed several classification scenarios and found that the source is most likely either a very peculiar BL Lac object or a CSO. The $\gamma$-ray brightness is well explained if PMN J1603-4904 is indeed a blazar, though the broadband emission and the mas-scale structure are very unusual. These spectral and structural features, atypical for a blazar, are accounted for by the alternative classification as a misaligned young radio source, possibly in a host galaxy with starburst activity.

If it is confirmed that this source is indeed not a BL Lac, it will add to the class of misaligned $\gamma$-ray bright sources. Only a few such sources are known and PMN J1603-4904 would be the first CSO in this class (Abdo et al. 2010e). The confirmed detection of a $\gamma$-ray bright CSO would challenge current jet emission models attempting to explain the high-energy spectral component with high beaming factors and would help to determine the region from where $\gamma$-rays are emitted. Hence, PMN J1603-4904 would be an important object to study the emission of highenergy photons in these misaligned sources and to address the open question of whether their $\gamma$-ray brightness is due to a beamed jet, lobe emission, or a combination of both.

TANAMI observations are ongoing, and with additional VLBI epochs, the kinematic analysis will be better constrained. Low resolution radio observations will be made at lower frequencies to better constrain the radio spectrum.

We will be attempting deeper photometric observations in the NIR (where the effects of Galactic extinction would be less severe) in order to establish the point source counterpart and obtain a spectrum further constraining the redshift. With additional $\mathrm{X}$-ray and optical observations, we will be able to better model and constrain the broadband SED.

Acknowledgements. The Australian Long Baseline Array and the Australia Telescope Compact Array are part of the Australia Telescope National Facility which is funded by the Commonwealth of Australia for operation as a National Facility managed by CSIRO. This research was funded in part by NASA through Fermi Guest Investigator grants NNH09ZDA001N and NNH10ZDA001N. It was supported by an appointment to the NASA Post-doctoral Program at the Goddard Space Flight Center, administered by Oak Ridge Associated Universities through a contract with NASA. It is based on observations obtained at the Gemini Observatory (Program ID: GS-2013A-Q-80), which is operated by the Association of Universities for Research in Astronomy, Inc., under a cooperative agreement with the NSF on behalf of the Gemini partnership: the National Science Foundation (United States), the National Research Council (Canada), CONICYT (Chile), the Australian Research Council (Australia), Ministério da Ciência, Tecnologia e Inovação (Brazil) and Ministerio de Ciencia, Tecnología e Innovación Productiva (Argentina). This research has made use of data from the NASA/IPAC Extragalactic Database (NED), operated by the Jet Propulsion Laboratory, California Institute of Technology, under contract with the National Aeronautics and Space Administration; and the SIMBAD database (operated at the CDS, Strasbourg, France). C.M. acknowledges the support of the "Studienstiftung des Deutschen Volkes". E.R. was partially supported 
by Spanish MINECO projects AYA2009-13036-C02-02 and AYA2012-38491C02-01 and by the Generalitat Valenciana project PROMETEO/2009/104, as well as by the COST MP0905 action "Black Holes in a Violent Universe". R.S. acknowledges support from Deutsche Forschungsgemeinschaft grant DFG WI1860/10-1. We thank I. Donnarumma for the useful discussion about starburst SEDs, R. D'Abrusco for performing checks on the corrected WISE data, and J. E. Davis for the development of the slxfig module used to prepare all figures in this work. We thank the referee for helpful comments and suggestions.

\section{References}

Abdo, A. A., Ackermann, M., Ajello, M., et al. 2009, ApJ, 700, 597 Abdo, A. A., Ackermann, M., Agudo, I., et al. 2010a, ApJ, 716, 30 Abdo, A. A., Ackermann, M., Ajello, M., et al. 2010b, ApJS, 188, 405 Abdo, A. A., Ackermann, M., Ajello, M., et al. 2010c, ApJ, 722, 520 Abdo, A. A., Ackermann, M., Ajello, M., et al. 2010d, ApJ, 709, L152 Abdo, A. A., Ackermann, M., Ajello, M., et al. 2010e, ApJ, 720, 912 Acciari, V. A., Aliu, E., Arlen, T., et al. 2009, Nature, 462, 770

Acero, F., Aharonian, F., Akhperjanian, A. G., et al. 2009, Science, 326, 1080 Ackermann, M., Ajello, M., Allafort, A., et al. 2011, ApJ, 743, 171 Ackermann, M., Ajello, M., Allafort, A., et al. 2012, ApJ, 755, 164 Ackermann, M., Ajello, M., Allafort, A., et al. 2013, ApJS, 209, 34 An, T., Wu, F., Yang, J., et al. 2012, ApJS, 198, 5

Antonucci, R. 1993, ARA\&A, 31, 473

Baum, S. A., O’Dea, C. P., Murphy, D. W., \& de Bruyn, A. G. 1990, A\&A, 232, 19

Bicknell, G. V., Dopita, M. A., \& O’Dea, C. P. O. 1997, ApJ, 485, 112 Biggs, A. D., Browne, I. W. A., Helbig, P., et al. 1999, MNRAS, 304, 349 Böck, M. 2012, Ph.D. Thesis, Friedrich-Alexander-Universität, ErlangenNürnberg, Germany

Burrows, D. N., Hill, J. E., Nousek, J. A., et al. 2005, Space Sci. Rev., 120, 165 Carvalho, J. C. 1998, A\&A, 329, 845

Chang, C.-S. 2010, Ph.D. Thesis, Max-Planck-Institut für Radioastronomie, Bonn, Germany

Chen, P. S., \& Shan, H. G. 2011, ApJ, 732, 22

D’Abrusco, R., Massaro, F., Paggi, A., et al. 2013, ApJS, 206, 12 Deller, A. T., Tingay, S. J., Bailes, M., \& West, C. 2007, PASP, 119, 318 Deller, A. T., Brisken, W. F., Phillips, C. J., et al. 2011, PASP, 123, 275 Dermer, C. D., Finke, J. D., Krug, H., \& Böttcher, M. 2009, ApJ, 692, 32 Dicken, D., Tadhunter, C., Axon, D., et al. 2012, ApJ, 745, 172

Donato, D., Ghisellini, G., Tagliaferri, G., \& Fossati, G. 2001, A\&A, 375, 739

Edwards, P. G., \& Tingay, S. J. 2004, A\&A, 424, 91

Elbaz, D., Dickinson, M., Hwang, H. S., et al. 2011, A\&A, 533, A119

Fanaroff, B. L., \& Riley, J. M. 1974, MNRAS, 167, 31P

Fanti, C., Fanti, R., Dallacasa, D., et al. 1995, A\&A, 302, 317

Fassnacht, C. D., \& Taylor, G. B. 2001, AJ, 122, 1661

Finke, J. D. 2013, ApJ, 763, 134

Fitzpatrick, E. L. 1999, PASP, 111, 63

Frail, D. A., \& Scharringhausen, B. R. 1997, ApJ, 480, 364

Gaensler, B. M., \& Slane, P. O. 2006, ARA\&A, 44, 17

Gehrels, N., Chincarini, G., Giommi, P., et al. 2004, ApJ, 611, 1005

Ghisellini, G., Tavecchio, F., Foschini, L., et al. 2010, MNRAS, 402, 497

Giommi, P., Polenta, G., Lähteenmäki, A., et al. 2012, A\&A, 541, A160

Giroletti, M., Giovannini, G., Taylor, G. B., \& Falomo, R. 2004, ApJ, 613, 752

Gregory, P. C., Vavasour, J. D., Scott, W. K., \& Condon, J. J. 1994, ApJS, 90, 173

Griffith, M. R., \& Wright, A. E. 1993, AJ, 105, 1666

Griffith, M. R., Wright, A. E., Burke, B. F., \& Ekers, R. D. 1994, ApJS, 90, 179 Högbom, J. A. 1974, A\&AS, 15, 417

Hovatta, T., Tornikoski, M., Lainela, M., et al. 2007, A\&A, 469, 899

Hovatta, T., Lister, M. L., Kovalev, Y. Y., Pushkarev, A. B., \& Savolainen, T. 2010, Int. J. Mod. Phys., 19, 943

Kadler, M. 2005, Ph.D. Thesis, Rheinische Friedrich-Wilhelms-Universität, Bonn, Germany

Kalberla, P. M. W., Burton, W. B., Hartmann, D., et al. 2005, A\&A, 440, 775

Kellermann, K. I., Lister, M. L., Homan, D. C., et al. 2004, ApJ, 609, 539

Kharb, P., Lister, M. L., \& Cooper, N. J. 2010, ApJ, 710, 764
Kino, M., \& Asano, K. 2011, MNRAS, 412, L20

Kino, M., Kawakatu, N., \& Ito, H. 2007, MNRAS, 376, 1630

Kino, M., Ito, H., Kawakatu, N., \& Nagai, H. 2009, MNRAS, 395, L43

Kovalev, Y. Y., Kellermann, K. I., Lister, M. L., et al. 2005, AJ, 130, 2473

Lenain, J.-P., Ricci, C., Türler, M., Dorner, D., \& Walter, R. 2010, A\&A, 524, A72

Linford, J. D., Taylor, G. B., Romani, R. W., et al. 2011, ApJ, 726, 16

Lira, P., Videla, L., Wu, Y., et al. 2013, ApJ, 764, 159

Lister, M. L., \& Homan, D. C. 2005, AJ, 130, 1389

Lister, M. L., Cohen, M. H., Homan, D. C., et al. 2009, AJ, 138, 1874

Lister, M. L., Aller, M., Aller, H., et al. 2011, ApJ, 742, 27

Malmrose, M. P., Marscher, A. P., Jorstad, S. G., Nikutta, R., \& Elitzur, M. 2011, ApJ, 732, 116

Massardi, M., Ekers, R. D., Murphy, T., et al. 2011, MNRAS, 412, 318

Massaro, E., Perri, M., Giommi, P., \& Nesci, R. 2004, A\&A, 413, 489

Massaro, F., D’Abrusco, R., Ajello, M., Grindlay, J. E., \& Smith, H. A. 2011, ApJ, 740, L48

Massaro, F., D’Abrusco, R., Tosti, G., et al. 2012, ApJ, 752, 61

McConville, W., Ostorero, L., Moderski, R., et al. 2011, ApJ, 738, 148

Müller, C., Böck, M., Wilms, J., et al. 2012, in Proc. Fermi \& Jansky: Our Evolving Understanding of AGN, eds. R. Ojha, D. Thompson, \& C. Dermer, eConf C1111101 [arXiv: 1205.1371]

Müller, C., Krauss, F., Kadler, M., et al. 2013, in 11th European VLBI Network Symposium \& Users Meeting, Proc. Science [arXiv: 1301.4384]

Murphy, T., Mauch, T., Green, A., et al. 2007, MNRAS, 382, 382

Murphy, T., Sadler, E. M., Ekers, R. D., et al. 2010, MNRAS, 402, 2403

Nolan, P. L., Abdo, A. A., Ackermann, M., et al. 2012, ApJS, 199, 31

Nowak, M. A., Neilsen, J., Markoff, S. B., et al. 2012, ApJ, 759, 95

O’Dea, C. P. 1998, PASP, 110, 493

Ojha, R., Kadler, M., Böck, M., et al. 2010, A\&A, 519, A45

Orienti, M., Dallacasa, D., Giovannini, G., Giroletti, M., \& D’Ammando, F. 2011, in Proc. 2011 Fermi Symposium, eConf C110509 [arXiv: 1111. 1185] Owsianik, I., \& Conway, J. E. 1998, A\&A, 337, 69

Peck, A. B., \& Taylor, G. B. 2000, ApJ, 534, 90

Piner, B. G., Pant, N., \& Edwards, P. G. 2010, ApJ, 723, 1150

Plotkin, R. M., Anderson, S. F., Brandt, W. N., et al. 2012, ApJ, 745, L27

Potter, W. J., \& Cotter, G. 2013, MNRAS, 431, 1840

Predehl, P., \& Schmitt, J. H. M. M. 1995, A\&A, 293, 889

Readhead, A. C. S., Taylor, G. B., Pearson, T. J., \& Wilkinson, P. N. 1996, ApJ, 460, 634

Roming, P. W. A., Kennedy, T. E., Mason, K. O., et al. 2005, Space Sci. Rev., 120,95

Shaw, M. S., Romani, R. W., Cotter, G., et al. 2013, ApJ, 764, 135

Shepherd, M. C. 1997, in Astronomical Data Analysis Software and Systems VI, eds. G. Hunt, \& H. Payne, ASP Conf. Ser., 125, 77

Skrutskie, M. F., Cutri, R. M., Stiening, R., et al. 2006, AJ, 131, 1163

Sokolovsky, K. V., Kovalev, Y. Y., Pushkarev, A. B., Mimica, P., \& Perucho, M. 2011, A\&A, 535, A24

Stawarz, L., Ostorero, L., Begelman, M. C., et al. 2008, ApJ, 680, 911

Stevens, J., Edwards, P. G., Ojha, R., et al. 2012, in Proc. Fermi \& Jansky: Our Evolving Understanding of AGN, eds. R. Ojha, D. Thompson, \& C. Dermer, eConf C1111101 [arXiv: 1205.2403]

Tadhunter, C., Holt, J., González Delgado, R., et al. 2011, MNRAS, 412, 960

Taylor, G. B., Readhead, A. C. S., \& Pearson, T. J. 1996, ApJ, 463, 95

Taylor, G. B., Fassnacht, C. D., Sjouwerman, L. O., et al. 2005, ApJS, 159, 27

Taylor, G. B., Charlot, P., Vermeulen, R. C., \& Pradel, N. 2009, ApJ, 698, 1282

Tingay, S. J., Edwards, P. G., \& Tzioumis, A. K. 2003, MNRAS, 346, 327

Urry, C. M., \& Padovani, P. 1995, PASP, 107, 803

Vermeulen, R. C., Ros, E., Kellermann, K. I., et al. 2003, A\&A, 401, 113

Verner, D. A., Ferland, G. J., Korista, K. T., \& Yakovlev, D. G. 1996, ApJ, 465, 487

Wilkinson, P. N., Henstock, D. R., Browne, I. W., et al. 2001, Phys. Rev. Lett., 86,584

Wilms, J., Allen, A., \& McCray, R. 2000, ApJ, 542, 914

Wilms, J., Juett, A. M., Schulz, N. S., \& Nowak, M. A. 2012, available http: // pulsar.sternwarte.uni-erlangen.de/wilms/research/tbabs/

Wright, E. L., Eisenhardt, P. R. M., Mainzer, A. K., et al. 2010, AJ, 140, 1868 